\title{
Bulimia Nervosa: Revisão da Literatura
}

\author{
Rita Aparecida Romaro ${ }^{1}$ \\ Fabiana Midori Itokazu \\ Universidade São Francisco
}

\begin{abstract}
Resumo
O presente estudo objetivou revisar a produção científica referente à bulimia nervosa, classificando-a em três dimensões de análise: países relacionados à pesquisa na área; periódicos de indexação; delineamentos e objetivos da pesquisa. A amostra foi composta de 200 abstracts de periódicos indexados nas bases de dados LILACS e MEDLINE, entre 1986 e outubro/2000, utilizando-se o descritor bulimia nervosa. Os Estados Unidos (43,5\%) e o Brasil (12,5\%) são os países com maior número de publicações. Os artigos estão distribuídos em 100 periódicos diferentes. O delineamento empírico predominou (75\%), com destaque para o procedimento de comparação de grupos (63,5\%), com a população adulta, predominado os estudos referentes às questões diagnósticas (24\%), psicodinâmicas (21,5\%) e aos aspectos ligados ao tratamento (17\%). Os dados encontrados sugerem um interesse crescente pelo assunto nos dois últimos anos, provavelmente pela maior incidência da doença, necessitandose de mais pesquisas que associem as influências sociais e os aspectos preventivos.

Palavras-chave: Distúrbio alimentar; bulimia nervosa; revisão da literatura; produção científica.
\end{abstract}

\section{Bulimia Nervosa: Literature Review}

\begin{abstract}
The aim of this paper was to revise the scientific production related to bulimia nervosa. It was classified in three dimensions of analysis: countries related to research in the area; indexed publications and designs and aims of the research. The sample comprised 200 abstracts of publications indexed in the LILACS and MEDLINE databases, between 1986 and october/2000, using the key word bulimia nervosa. The United States $(43,5 \%)$ and Brazil $(12,5 \%)$ are the countries having the largest number of publications. The articles are distributed among 100 different journals. The empirical design predominated (75\%), with a greater emphasis on the procedure of group comparisons $(63,5 \%)$, with adult population. Studies on diagnosis issues $(24 \%)$ psychodynamic issues $(21,5 \%)$ and aspects linked to treatment $(17 \%)$. The data found suggest an increasing interest in the subject over the past two years, probably due to the higher incidence of the disease. More research is needed, associating social influences and preventive aspects.

Keywords: Eating disorder; bulimia nervosa; literature review; scientific production.
\end{abstract}

A mídia e o imaginário coletivo parecem estabelecer uma estreita relação entre a forma do corpo e a saúde, como se todos os regimes, dietas, exercícios físicos pudessem ser utilizados no sentido do indivíduo cuidarse melhor, tornando-se mais saudável. No entanto, na última década, os casos de transtornos alimentares proliferaram, principalmente os quadros de bulimia, anorexia nervosa e obesidade, criando-se ambulatórios de saúde específicos para o tratamento médico e psicológico.

Nos últimos anos a valorização excessiva da forma e do peso do corpo tem levado muitas pessoas, principalmente mulheres, a verdadeiros sacrifícios que podem comprometer a saúde, como dietas radicais (que proliferam a cada semana) e exercícios físicos em excesso, com o intuito de conseguirem chegar ao corpo ideal.

\footnotetext{
${ }^{1}$ Endereço para correspondência: Av. Aclimação, 439/141, 01531 001, São Paulo, SP. Fone/fax (11) 3208-9661. E-mail: romarorita@uol.com.br
}

Todo esse esforço pode acabar resultando na bulimia nervosa, uma doença que consiste na compulsão periódica de alimentos, seguida da utilização de estratégias para 'eliminar' as calorias ingeridas, podendo ocorrer por métodos purgativos (auto-indução de vômitos ou uso indiscriminado de laxantes, diuréticos ou enemas) e não purgativos (jejuns e exercícios físicos excessivos).

A pessoa que desenvolve o quadro de bulimia nervosa, em geral, valoriza muito a forma do corpo e o peso, possuindo uma percepção física distorcida e dificuldade em identificar as emoções. Apresenta uma baixa autoestima, um nível elevado de ansiedade, um baixo limiar à frustração e um prejuízo no controle dos impulsos. Em sua insegurança, elege padrões de beleza muito altos, praticamente inatingíveis, na tentativa de corresponder à tendência da sociedade em eleger a magreza como símbolo de sucesso e beleza. Ao perceber que não atingiu suas metas, sente-se deprimida, fracassada e retorna à compulsão, com conseqüente culpa e depressão (Behar, 1994). 
Comumente sente vergonha de seu transtorno e tenta primeiramente negar, depois ocultar, dissimular os sintomas, apresentando dificuldades marcadas no âmbito dos relacionamentos interpessoais. Pode ainda apresentar tendências perfeccionistas, dificuldade em assumir compromissos e responsabilidades e uma organização sexual, em geral, conturbada e confusa, sendo comum a promiscuidade.

A bulimia nervosa é um transtorno da alimentação que possui enquanto características fundamentais: episódios recorrentes de compulsões periódicas (ingestão de uma grande quantidade de alimentos, em um espaço curto de tempo, que dura em torno de 2 horas) e um sentimento de falta de controle sobre o comportamento alimentar durante o episódio; comportamento compensatório inadequado recorrente, com o fim de prevenir o aumento de peso, como a auto-indução de vômito, uso indevido de laxantes, diuréticos, enemas ou outros medicamentos, jejuns ou exercícios excessivos; ocorrência de compulsões, no mínimo, duas vezes por semana, no espaço de 3 meses; auto-avaliação indevidamente influenciada pela forma e peso do corpo; o transtorno não ocorrer exclusivamente durante episódios de anorexia nervosa (DSM-IV, 1995).

As compulsões apresentam-se associadas a estados de humor disfóricos, como depressão, situações negativas ou provocadoras de stress. Também são observados sentimentos relacionados à perda ou à rejeição, baixa autoestima, insegurança, restrição alimentar devido a dietas, sentimentos relacionados ao peso e a forma do corpo (Behar, 1994; De Conti, Moreno \& Cordas, 1995).

De acordo com estudos apontados no DSM-IV (1995) encontra-se associação da bulimia nervosa, com transtornos de personalidade, principalmente o borderline, transtorno de ansiedade, transtorno obsessivo-compulsivo, sintomas depressivos ou transtornos de humor como a depressão maior e a distimia, abuso ou dependência de substâncias (álcool, estimulantes). Segundo a Sociedade Brasileira de Psiquiatria (SBP, 1993), as pessoas portadoras dos transtornos acima citados apresentam tendências impulsivas que podem influenciar um comportamento suicida, sendo que o índice de mortalidade nos transtornos alimentares é de $10 \%$.

A bulimia nervosa é considerada como um transtorno alimentar específico desde 1980, com a publicação do DSM-III e a cada ano observa-se um maior interesse de estudiosos por diferentes facetas desse transtorno. O que estimularia essa investigação? A maior incidência do transtorno? A necessidade de um refinamento na precisão diagnóstica? Em que direção se desenvolvem os estudos?
Tomando-se por base as questões acima formuladas, o presente estudo objetivou revisar a produção científica referente à bulimia nervosa, a partir da década de 1980 , classificando-a em três dimensões de análise: países relacionados à publicação de pesquisa na área; periódicos de indexação; delineamentos e objetivos visados pela pesquisa.

\section{Método}

Compreendeu a análise de 200 abstracts de periódicos indexados na base de dados LILACS (71 resumos) e MEDLINE (183 resumos), entre 1986 e outubro/2000, utilizando-se o descritor bulimia nervosa ${ }^{2}$. Foram encontrados um total de 252 resumos, no entanto, apenas $200(78,7 \%)$ traziam dados completos referentes ao local de publicação e ao delineamento de pesquisa empregado. O tratamento dos dados foi realizado por meio da análise das freqüências percentuais.

\section{Resultados e Discussão}

A primeira dimensão de análise refere-se ao local e ano de publicação dos artigos, desde 1986. Os resultados, em percentagem encontram-se na Tabela 1.

Os dois primeiros artigos encontrados remontam a 1986, publicados no Chile. No Brasil as publicações iniciaram-se em 1988, sendo contínuas até 1999. O Chile e o Brasil detiveram o monopólio das publicações até o ano de 1994, sendo que até o ano de 1997 as publicações da América do Sul eram as únicas referências indexadas na base de dados LILACS (com exceção de um trabalho apresentado em um periódico mexicano em 1995), não tendo sido encontradas referências de publicações no ano 2000 nessa base de dados. Dos 21\% artigos publicados na América do Sul, o Brasil responde por 12,5\%. As publicações na América do Norte (45,5\%) concentramse de 1998 a 2000, sendo lideradas pelos Estados Unidos (43,5\%). A Europa responde por $30 \%$ das publicações científicas, com destaque para o Reino Unido ( $8 \%$ ), Itália (6,5\%) e Holanda (4,5\%). Os estudos publicados em 1999 e 2000 correspondem a $77 \%$ do total. As publicações na Ásia e na Oceania são pouco expressivas e não foram encontrados referências de artigos publicados no continente africano.

A segunda dimensão de análise refere-se aos periódicos de indexação. Os 200 artigos foram publicados em 100

\footnotetext{
${ }^{2}$ A relação dos trabalhos consultados pode ser obtida com os autores, via e-mail.
} 
Tabela 1

Distribuição Percentual dos Artigos por Ano e Local de Publicação

\begin{tabular}{|c|c|c|c|c|c|c|c|c|c|c|c|c|c|c|c|}
\hline Países & 86 & 88 & 89 & 90 & 91 & 92 & 93 & 94 & 95 & 96 & 97 & 98 & 99 & 00 & Total \\
\hline Brasil & & 1 & 0,5 & 0,5 & 0,5 & 0,5 & 1 & 1 & 0,5 & 1 & 1 & 3 & 2 & & 12,5 \\
\hline Chile & 1 & & & 0,5 & 0,5 & & & 1 & 0,5 & 1,5 & 1,5 & & & & 6,5 \\
\hline Argentina & & & & & & & & & & & 0,5 & 0,5 & & & 1 \\
\hline Colômbia & & & & & & & & & 0,5 & & 0,5 & & & & 1 \\
\hline EUA & & & & & & & & & & & & 0,5 & 9,5 & 33,5 & 43,5 \\
\hline Canadá & & & & & & & & & & & & & 0,5 & 0,5 & 1 \\
\hline México & & & & & & & & & 0,5 & & & & & 0,5 & 1 \\
\hline Itália & & & & & & & & & & & & 2,5 & 3,5 & 0,5 & 6,5 \\
\hline R. Unido & & & & & & & & & & & & 0,5 & 3 & 4,5 & 8 \\
\hline França & & & & & & & & & & & & & 1,5 & 0,5 & 2 \\
\hline Alemanha & & & & & & & & & & & & & 0,5 & 1 & 1,5 \\
\hline Espanha & & & & & & & & & & & & & 1 & 0,5 & 1,5 \\
\hline Holanda & & & & & & & & & & & & & 1,5 & 3 & 4,5 \\
\hline Suíça & & & & & & & & & & & & & 1 & 1,5 & 2,5 \\
\hline Áustria & & & & & & & & & & & & & 0,5 & & 0,5 \\
\hline Dinamarca & & & & & & & & & & & & & 1 & 1,5 & 2,5 \\
\hline Suécia & & & & & & & & & & & & & & 0,5 & 0,5 \\
\hline Índia & & & & & & & & & & & & & & 0,5 & 0,5 \\
\hline Israel & & & & & & & & & & & & & & 0,5 & 0,5 \\
\hline Japão & & & & & & & & & & & & & & 0,5 & 0,5 \\
\hline Austrália & & & & & & & & & & & & & 1 & 1 & 2 \\
\hline Total & 1 & 1 & 0,5 & 1 & 1 & 0,5 & 1 & 2 & 2 & 2,5 & 3,5 & 7 & 26,5 & 50,5 & 100 \\
\hline
\end{tabular}

Tabela 2

Distribuição das Publicações por Periódicos Indexados

\begin{tabular}{llc}
\hline Periódicos & $f$ & $\%$ \\
\hline Internacional Journal Eat Disorders (EUA) & 37 & 18,5 \\
Eat Weight Disord (Itália) & 12 & 6 \\
Jornal Brasileiro de Psiquiatria (Brasil) & 8 & 4 \\
Acta Psychiatrica Scandinavica (Dinamarca) & 5 & 2,5 \\
American Journal of Psychiatry (EUA) & 5 & 2,5 \\
Psychiatry Research (Holanda) & 4 & 2 \\
Revista Child Neuropsiquiatric (Chile) & 4 & 2 \\
Revista ABP-APAL (Brasil) & 4 & 2 \\
Journal of Consulting and Clinical Psychology (EUA) & 4 & 2 \\
Journal of Clinical Psychiatry (EUA) & 3 & 1,5 \\
Revista de Psiquiatria (Chile) & 3 & 1,5 \\
Biological Psychiatric (EUA) & 3 & 1,5 \\
Revista de Psiquiatria (RS - Brasil) & 3 & 1,5 \\
Revista de Psiquiatria Clínica (SP-Brasil) & 3 & 1,5 \\
European Psychiatry (França) & 3 & 1,5 \\
Outras (1 ou 2 artigos) & 99 & 49,5 \\
\hline Total & 200 & 100 \\
\hline
\end{tabular}


periódicos diferentes, sendo que em $72 \%$ desses encontrase somente uma publicação sobre o tema. Em ordem crescente de publicação encontra-se a seguinte percentagem de artigos em um mesmo periódico: com duas publicações $14 \%$; com três publicações $5 \%$; com quatro publicações $4 \%$; com cinco publicações $2 \%$; com oito, 12 e 37 publicações 1\% cada um.

Nos Estados Unidos, os 87 artigos publicados distribuem-se em 35 periódicos, no Brasil, as 25 publicações distribuem-se em 10 periódicos, na Itália em dois. As 16 publicações do Reino Unido são encontradas em 13 periódicos e as 13 publicações chilenas em cinco periódicos. Todas as publicações da Holanda encontramse em um mesmo periódico. A Tabela 2 apresenta os periódicos que publicaram mais de dois artigos sobre o tema no período de 1986 a 2000.

Entre os periódicos com mais de dois artigos publicados sobre bulimia nervosa, cinco são norteamericanos, responsáveis pelo maior percentual de publicações $(26 \%)$; quatro são brasileiros $(9 \%)$, um italiano $(6 \%)$ e dois chilenos $(3,5 \%)$. Destacam-se também periódicos dinamarqueses $(2,5 \%)$, holandeses $(2 \%)$, e franceses $(1,5 \%)$.

A terceira dimensão de análise abarca os dois delineamentos empregados nas pesquisas. O conceitual, quando refere-se predominantemente à revisões literárias; e, o experimental, quando os trabalhos apresentam um procedimento empírico de coleta de dados, podendo ser por meio de comparação de grupos, estudo evolutivo ou estudo de caso. $\mathrm{Na}$ Tabela 3 são identificados os delineamentos de pesquisa dos trabalhos revisados, ressaltando-se a idade dos participantes.

Nos estudos revisados $25 \%$ empregaram um delineamento conceitual de revisão de literatura e 75\% um delineamento empírico, sendo que desses $63,5 \%$ referiamse a procedimentos de comparação de grupos, $6 \%$ ao de estudo de caso e 5,5\% ao estudo evolutivo. Nos dois delineamentos de pesquisa empregados predominaram os estudos com a população adulta $(93 \%)$, sendo $5 \%$ desses trabalhos realizados com adolescentes e jovens adultos. Somente com os adolescentes encontrou-se 13 estudos $(6,5 \%)$ e com a população infantil apenas um estudo $(0,5 \%)$. A pesquisa exploratória caracterizada pelos delineamentos de revisão da literatura e estudo de caso, foi empregada em $31 \%$ das pesquisas, evidenciando a complexidade e a abrangência de um tema que a partir de 1998 tem despertado ainda mais o interesse dos pesquisadores, principalmente dos norte-americanos.

Apesar da bulimia nervosa iniciar-se comumente na adolescência, ainda são poucos os estudos com essa faixa etária, embora alguns abranjam a adolescência e o início da fase adulta, buscando índices precoces de risco para o desenvolvimento da doença. O predomínio de estudos com participantes adultos talvez deva-se ao fato da bulimia nervosa iniciar-se no final da adolescência ou início da fase adulta, sendo o tratamento em geral procurado já na idade adulta e a maioria das pesquisas realizadas referirse a pacientes em tratamento. Na Tabela 4 são apresentados os estudos de acordo com os objetivos predominantes e o delineamento de pesquisa empregado.

Etiologicamente as pesquisas ressaltam a complexa interação entre os fatores bioquímicos, socioculturais e psicológicos. A influência genética tem sido investigada, encontrando-se uma maior incidência de bulimia entre os gêmeos monozigóticos. Um outro aspecto estudado é a presença de depressão e de distúrbios alimentares nas mães de bulímicos. Os estudos que ocupam-se da etiologia da doença somam $11,5 \%$, com destaque aos aspectos bioquímicos $(10,5 \%)$, com predomínio da metodologia de comparação de grupos e com destaque para os efeitos da regulação anormal da serotonina, distúrbios nos sistemas neuroendócrino, neurotransmissor e neuropéptico.

Tabela 3

Delineamentos de Pesquisa Empregados e Faixa Etária da População Estudada

\begin{tabular}{|c|c|c|c|c|c|c|c|c|c|c|}
\hline \multirow{3}{*}{$\begin{array}{l}\text { Delineamento } \\
\text { / faixa etária }\end{array}$} & \multirow{2}{*}{\multicolumn{2}{|c|}{ Conceitual }} & \multicolumn{6}{|c|}{ Experimental } & \multicolumn{2}{|c|}{ Total } \\
\hline & & & \multicolumn{2}{|c|}{$\begin{array}{l}\text { Estudo de } \\
\text { caso }\end{array}$} & \multicolumn{2}{|c|}{$\begin{array}{l}\text { Estudo } \\
\text { evolutivo }\end{array}$} & \multicolumn{2}{|c|}{$\begin{array}{c}\text { Comparação } \\
\text { de grupos }\end{array}$} & \multirow[t]{2}{*}{$f$} & \multirow[t]{2}{*}{$\%$} \\
\hline & $f$ & $\%$ & $f$ & $\%$ & $f$ & $\%$ & $f$ & $\%$ & & \\
\hline Criança & & & 1 & 0,5 & & & & & 1 & 0,5 \\
\hline Adolescente & 2 & 1 & & & & & 11 & 5,5 & 13 & 6,5 \\
\hline $\begin{array}{l}\text { Adolescente e } \\
\text { Adultos }\end{array}$ & & & 1 & 0,5 & 1 & 0,5 & 8 & 4 & 10 & 5 \\
\hline Adulto & 48 & 24 & 10 & 5 & 10 & 5 & 108 & 54 & 176 & 88 \\
\hline Total & 50 & 25 & 12 & 6 & 11 & 5,5 & 127 & 63,5 & 200 & 100 \\
\hline
\end{tabular}


Tabela 4

Objetivos dos Estudos Revisados - Revisão da Literatura e Pesquisa Experimental

\begin{tabular}{lcccccc}
\hline Objetivos/ Delineamento & \multicolumn{2}{c}{ Revisão da literatura } & \multicolumn{2}{c}{ Pesquisa experimental } & \multicolumn{2}{c}{ Total } \\
& $f$ & $\%$ & $f$ & $\%$ & $f$ & $\%$ \\
\hline Etiologia & 1 & 0,5 & 1 & 0,5 & 2 & 1 \\
$\quad$ Bioquímica & 7 & 3,5 & 14 & 7 & 21 & 10,5 \\
Curso da doença & & & 2 & 1 & 2 & 1 \\
Complicações médicas & 2 & 1 & 6 & 3 & 8 & 4 \\
Transtornos associados & 1 & 0,5 & 18 & 9 & 19 & 9,5 \\
Diagnóstico & 20 & 10 & 38 & 19 & 58 & 29 \\
Compreensão do quadro & & & & & & \\
$\quad$ Psicodinâmica & 7 & 3,5 & 36 & 18 & 43 & 21,5 \\
$\quad$ Familiar & 2 & 1 & 5 & 2,5 & 7 & 3,5 \\
$\quad$ Influências sociais & & & 4 & 2 & 4 & 2 \\
$\quad$ Tratamento & & & & & & \\
$\quad$ Parmacológico & 4 & 2 & 8 & 4 & 12 & 6 \\
$\quad$ Outroterápico & 1 & 0,5 & 5 & 2,5 & 6 & 3 \\
Prevenção & 4 & 2 & 12 & 6 & 16 & 8 \\
Total & 1 & 0,5 & 1 & 0,5 & 2 & 1 \\
\hline
\end{tabular}

Os estudos que objetivam compreender aspectos da bulimia nervosa relacionados ao curso e às complicações médicas perfazem um total de 5\%. São enfatizadas as pesquisas abarcando as complicações médicas (4\%), principalmente as associadas aos distúrbios endócrinos, como a diabetes tipo I, que pode conduzir a um deficitário controle metabólico e ao aumento do risco para complicações microvasculares. Transtornos do aparelho genital e manifestações cutâneas também são estudados.

As questões diagnósticas (29\%) e os transtornos associados (9,5\%) aparecem em 38,5\% dos estudos, com um predomínio do emprego da metodologia de comparação de grupos. A investigação diagnóstica objetiva tanto a detecção precoce dos indícios de bulimia quanto o estudo da composição da bulimia com os possíveis transtornos associados, como os transtornos afetivos, os transtornos de ansiedade, o transtorno de personalidade borderline, a anorexia nervosa, o 'comer compulsivo', entre outros. Na busca de diferenciação do quadro, várias escalas foram construídas e validadas, sendo amplamente empregadas, tais como: Eating Disorder Examination (EDE; Fairburn \& Cooper, 1993), Eating Attitudes Test (EAT-26; Gross, Rosen, Leitenberg \& Willmuth, 1986), Eating Disorder Inventory (EDI; Garner, Olmstedt \& Polivy, 1983; Nunes \& cols., 1994), Bulimic Investigatory Test Edinburgh (BITE; Cordás \& Hochgraf, 1993; Henderson \& Freeman, 1987), Beck Depression Inventory (BDI; Beck, Rush, Shaw \& Emery, 1979), e Minnesota Multiphasic Personality Inventory (MMPI; Hathaway \& McKinley, 1943).
A compreensão da doença do ponto de vista psicodinâmico e social é abordada em $27 \%$ dos estudos, com a seguinte distribuição: compreensão psicodinâmica $(21,5 \%)$, aspectos relacionados explicitamente à psicodinâmica familiar $(3,5 \%)$ e influências sociais $(2 \%)$. A compreensão dos aspectos psicodinâmicos, como a baixa auto-estima, a ansiedade, a culpa, as tendências perfeccionistas, a dificuldade no controle dos impulsos, o tipo de vínculo com a figura materna, o limiar de resistência ao stress podem também ser utilizada como auxiliar nas atuações preventivas e na detecção precoce da enfermidade.

As questões ligadas ao tratamento aparecem em 17\% dos estudos, distribuindo-se da seguinte forma: questões farmacológicas $(6 \%)$, destacando-se os efeitos do uso da fluoxetina; questões psicoterápicas $(3 \%)$ e questões envolvendo uma diversidade de outras modalidades de tratamento (8\%). As psicoterapias cognitivas e comportamentais são apontadas como as mais eficazes no tratamento do bulímco por possibilitarem a conscientização da gravidade do transtorno, viabilizando seu controle. A associação medicamentosa e psicoterápica tem possibilitado melhores resultados.

Os estudos preventivos ainda se mostram muito incipientes $(1 \%)$. Apesar do pouco conhecimento dos efeitos da bulimia nervosa a longo prazo, os estudos objetivando explorar o curso da doença são escassos, bem como os envolvendo as influências sociais e a prevenção, apesar da preocupação com esses aspectos serem apontados em grande parte dos estudos. 


\section{Considerações Finais}

Até o ano de 1998 eram poucos os estudos indexados nas bases de dados LILACS e MEDLINE que ocupavam-se de estudar a bulimia nervosa, no entanto, a partir dessa data diversos estudos começaram a proliferar, principalmente na Europa e nos Estados Unidos, visto a propagação da doença e sua difícil terapêutica. Encontramos na presente revisão tanto estudos de caráter exploratório, como as revisões de literatura e os estudos de caso, quanto estudos que visam a comparação entre grupos, predominando a faixa etária adulta, em função do próprio curso da doença, que tem seu início no final da adolescência e início da fase adulta.

Os artigos levantados no período de 1986 a 1990 revisam aspectos fisiológicos, patológicos e terapêuticos, buscando uma melhor precisão diagnóstica, e possíveis transtornos associados ou diagnósticos diferenciais. Alguns autores descrevem psicodinamicamente a pessoa bulímica, outros as relações familiares, de acordo com o referencial psicanalítico. A partir de 1991 são encontrados alguns trabalhos com enfoque bioquímico, com destaque para o papel da serotonina e a partir de 1993 trabalhos utilizando escalas como a Bulimic Investigatory Test Edinburgh (BITE), o Minnesota Multiphasic Personality Inventory (MMPI), Eating Attitudes Test (EAT-26) e o Eating Disorder Examination (EDE), buscando uma melhor caracterização e compreensão do quadro. Aspectos psicodinâmicos e evolutivos são abordados. Alguns estudos com a Beck Depression Inventory ressaltam os aspectos familiares da depressão e o tipo de vinculação familiar.

A partir de 1998, com o grande aumento das publicações, encontra-se o aprofundamento dos aspectos anteriormente estudados, com a construção e validação de escalas e preocupação com a eficácia das abordagens terapêuticas, com alguns estudos empregando o follow-up. As psicoterapias comportamental e cognitiva são apontadas como as mais eficazes, considerando-se que melhores resultados são obtidos com a associação das terapêuticas psicoterápica e medicamentosa, sendo enfatizado o uso da fluoxetina. A compreensão psicodinâmica começa a ser utilizada no sentido de detecção de riscos precoces para o desenvolvimento do transtorno. As influências sociais têm sido pouco estudadas, apesar de reconhecidas. Alguns estudos de prevalência com estudantes (adolescentes e jovens adultos) têm sido desenvolvidos apesar de ainda serem escassos os trabalhos preventivos.

\section{Referências}

Associação Americana de Psiquiatria (1995). Manual Diagnóstico e Estatístico de Transtornos Mentais- DSM IV. (4 ed.) (D. Batista, Trad.). Porto Alegre: Artes Médicas.

Behar, V. S. (1994). Abordagem psicoterápica do paciente com bulimia nervosa. Insight-psicoterapia, 43, 11-14.

Beck, A. T., Rush, A. J., Shaw, B. F. \& Emery, G. (1979). Cognitive therapy of depression. New York: Guilford Press.

Cordás, T. A. \& Hochgraf, P. B. (1993). O BITE. Instrumento para avaliação da Bulimia nervosa: Versão para o português. Jornal Brasileiro de Psiquiatria, 42, 141-144.

De Conti, F. N. B., Moreno, R. A. \& Cordas, T. A. (1995). Avaliação da bulimia em pacientes com transtorno do humor através da escala BITE: Resultados preliminares. Revista ABP-APAL, 17(2), 72-74.

Fairbaum, C. G. \& Cooper, Z. (1993). The eating disorders examination. (12 ed.). Em C. G. Fairbaum \& G. T. Wilson (Orgs.), Binge eating: Nature, assessment and treatment (pp. 160-192). New YorK: Guilford Press.

Garner, D. M., Olmstedt, M. P. \& Polivy, J. (1983). Development and validation of a multidimensional eating disorders inventory for anorexia and bulimia nervosa. International Journal of Eating Disorders, 2, 14-34.

Gross, J., Rosen, J. C., Leitenberg, H. \& Willmuth, M. E. (1986). Validity of the Eating Attitudes Test and the Eating Disorders Inventory in Bulimia Nervosa. Journal of Consultant and Clinical Psychology, 54, 875876.

Henderson, M. \& Freeman, C. P. (1987). A self-rating scale for bulimia. The BITE. British Journal of Psychiatry, 150, 18-24.

Nunes, M. A., Bagatini, L. F., Abuchaim, A. L., Kunz, D. R., Silva, J. A., Somenzi, L. \& Pinheiro, A.(1994). Distúrbio da conduta alimentar: Considerações sobre o Teste de Atitudes Alimentares (EAT). Revista ABP-APAL, 16(1), 7-10.

Sociedade Brasileira de Psiquiatria (SBP) (1993). Programa de Educação Sanitária. A década do cérebro: Transtornos Alimentares. Livreto traduzido e adaptado National Institute of Mental Health (1993). Eating Disorders (DHHS Publication No. NIH 93-3477).

\section{Sobre as autoras}

Rita Aparecida Romaro é Doutora em Psicologia Clínica pelo Instituto de Psicologia da Universidade de São Paulo. É Professora do programa de Pós-graduação em Avaliação Psicológica na Universidade São Francisco.

Fabiana Midori Itokazu é Psicóloga, graduada na Universidade São Francisco em 2000. 